\title{
1
}

\section{The 3 "A"s: Authentication, Authorization, Accounting}

For the road travelers in the United States, especially the parents who take their children in the family car on the long road trips, the letters AAA stand for a peace of mind. They feel that any time their car breaks down, they can call the number for the American Automobile Association and ask for roadside assistance. Even though this book is not about that sort of AAA, the 3 "A"s that we talk about here, when designed properly, can bring the same peace of mind to the network operator and its customers. Authentication, authorization, and accounting are three important blocks used in the construction of a network architecture that helps protect the network operator and its customers from fraud, attacks, inappropriate resource management, and loss of revenue.

In this chapter, we describe each of the "A"s in the AAA first as a separate topic, and then as a piece that interacts with the other "A"s in an effort to justify why all the 3 "A"s should be treated by the same framework and servers. At the end of the chapter, we provide a model for a generic AAA architecture.

\subsection{Authentication Concepts}

According to the dictionary, the word "authentic" refers to something that is not false, or a fake imitation, but is worthy of acceptance as a truth or a fact. From the times of early civilizations, where people have run 26 miles only to deliver a message and then fall over and die, to today, when information can travel across the globe in fractions of a minute with a mouse click, proof of authenticity is the first thing the receiver of a message checks.

Authentication consists of two acts: first, the act of providing proof of authenticity for the information that is being delivered or stored, and second, the act of verifying the proof of authenticity for the information that is being received or retrieved. In the early ages, an emperor would use his personal seal on his letters to provide assurance for the authenticity of the letter. The letter could then be carried by any messenger, whose identity was not important. The local lord would recognize the emperor seal and trust authenticity of the letter. He would

AAA and Network Security for Mobile Access: Radius, Diameter, EAP, PKI and IP Mobility

Madjid Nakhjiri and Mahsa Nakhjiri (C) 2005 John Wiley \& Sons, Ltd 
break the seal, read the letter, start an attack or collect taxes accordingly. In the days of digital information delivery, delivering proof of authenticity is equally important but poses its own challenges, as we will see.

The message delivery example above presents one type of authentication problem where authenticity of the information is important, while the identity of the messenger is not. However, in most of the cases, the identity of a person we are dealing with is an important factor in how we handle that interaction. When we go to a bank or through customs into a new country, we have to show identification to prove our identity. At first, the problem of identification does not seem to be related to the authentication. However, when one thinks about the possibility of a person lying about her identity or privileges, verification of authenticity of the provided identity becomes an authentication problem as well. Stating a name is typically not enough for identification, while showing a sort of identification issued by a trusted authority typically is. The acts of providing proof and verifying the authenticity of the identification presented are again the two acts of authentication.

Today, the two mentioned forms of authentications, i.e. providing information integrity and identity verification, are among the most fundamental security mechanisms required for providing access to network users and clients. In this introductory section, we provide a relatively short overview of various authentication concepts to allow the reader to understand the distinction between the constantly confused types of authentication. In Chapter 2, we will delve into more details of various authentication procedures.

\subsubsection{Client Authentication}

Client authentication means that a client wishing to gain access and connect to the network presents its identity along with a set of credentials. As proof of authencity for the presented identity. The credentials are then used by the network to verify that the identity actually belongs to the client.

We intentionally used the term client, since it can be interpreted both as a device as well as a human user, who is a consumer of a network service. For that reason, the client authentication needs to be further refined into two categories: user authentication and device authentication. Until recently, very few network security designs made a visible distinction between user authentication and device authentication. In the following we will explain the reason. Traditional architectures dealing with network access control could be divided into two categories:

- Architectures accommodating users that arrive at a fixed location, such as a local area network with fixed devices, such as data terminals, already connected to an infrastructure. The user needs to use its personal credentials to log into the network through a device (terminal), which itself typically resides in a computer room and is trusted through its wired connections. A good old world college campus terminal room scenario! The student simply trusts the network set up by the campus, as long as the college is an accredited one and the terminal is not asking for credit card numbers as login credentials! In this basic scenario, the distinction between device and user authentication although very clear for a human, is not important. The device is not authenticated at all. The user credential with a central server is the main criterion for allowing network access to the user.

- Architectures accommodating mobile users carrying their personal devices to gain access to a wide area network. A perfect example is cellular phone systems. The user registers 
with a cellular service provider and purchases a cellular phone that works with operator's network. The operator creates a set of credentials specific for the user. The cellular phone, that the user carries, is then programmed with such credentials to access the network. Many times, the user is unaware of these credentials and the actual process of authentication during connection establishment. The device is the entity that interacts with the network and presents the credentials needed to perform authentication. The idea is: since the user always carries the same cellular phone (as long as she is loyal to her service provider) no distinction between the user and device credentials has to be made. The downside is that if the device was lost, stolen, or even cloned, the rouge or unintended user could use the device to gain access to the network without having her real identity exposed, and this could go on until the legitimate user would report a lost or stolen phone or illegitimate entries in her monthly statement from the service provider.

With the proliferation of public local network providers, such as wireless hot spots serving passing customers, many sorts of vulnerabilities will appear at various corners of the architecture:

- The long-term customer-operator business and legal relationship no longer exists, which means the network operator and the user cannot trust each other as.

- The one-to-one mapping of user-device does not exist. Even if the operator could trust a user, the operator would not know what device the user may use every time they try to access the network. In other cases, such as in service organizations, government agencies, or police department, users that belong to a team can share their devices with each other.

Such refinements require more precise definition of the network usage and security policies that in turn means the architecture must be designed more carefully. The network operator may need to make sure that both device and user are authentic, possibly using separate processes. In some cases, the device may have to even be manufactured and configured with credentials for access to the network. (For instance, cable modems for cable Internet Service Provider (ISP) networks are produced this way.) In such cases, proper protection must be in place, so that the credentials on the device can not be tampered with. The network operator also needs to make sure the user presents accurate identity and proper credentials that can identify her at the time of use, so that the various users can be distinguished even when they are using a shared device.

Device authentication credentials can be certificates or cryptographic keys that are loaded in the devices either by the manufacturer in the factory or by the network operator at the time of service initiation. When designed properly and in a modular manner, the device authentication process should be transparent to the user. In fact, the user should not even be allowed to access device authentication credentials. On the other hand, user authentication credentials are personalized, typically given to the user in an out-of-band method. Examples are over a phone call by the user stating some secrets about her identity or through a face-to-face meeting after presentation of a driving license, company badge, and so on. Upon identification of the user, the system operator issues the authentication credentials for the user. The credentials must be carried by the user at all times either memorized (password) or in the form of a token such as a secure ID card, a certificate on some sort of cryptographic module. The user applies her authentication credentials on the device provided for access to the network to connect to the network. 
It should be noted that the security architecture may require both device authentication as well as user authentication in various steps of a network access process. An example would be the case of IP networks: in order to communicate to the IP network, the device needs to acquire an IP address. The IP address is not only an important resource for the network, but also allows the user to gain access to many other network services. Furthermore, the IP address can be used as a backdoor to launch active or passive attacks. Hence, IP address acquisition should be tightly controlled. From a security standpoint, it means a device needs to first authenticate itself to the network before being able to gain an IP address from the network. Once the device is authenticated, has gained IP address from the network, and is registered with various agents, it allows the user to present her credentials to the network and gain access to the services to which she is entitled. The latter brings another point and that is the user credentials may be used to determine authorization levels for the users based on their pre-configured service profile. We will discuss the topic of authorization later on.

\subsubsection{Message Authentication}

Device or user authentications deal with ensuring that the end points of the communications are legitimate and who they claim they are. Message authentication, on the other hand, ensures and verifies the integrity of the data at hand (remember the example of sealed letter from the emperor). When message authentication is performed, the receiver of the message can be sure that the information included in the message has been produced by a legitimate source and not been altered by other parties in transit. This is why message authentication is usually considered as a data integrity protection mechanism. Unlike device or user authentications that are typically performed at the beginning of a session and require their own messaging mechanism, message authentication may have to be done quite often during the session and for a variety of traffic, such as control messaging, important data packets, and sometimes even data session.

Note that the goal of data integrity protection is to prevent malicious and intended corruption of data by the so-called men in the middle (MITM), trying to tamper with the message contents. This is different from the information-theoretical codes and cyclic redundancy checks designed to mitigate the random and natural data corruptions caused by physical communications media imperfections. Aside from the cause of corruption being different, calculations required for message authentication for security protection is also different from its signal processing counter parts; an attacker can always alter the data and re-calculate the information-theoretical checks to lure the receiver, while the attacker cannot re-calculate the message authentication data added to the message, since message authentication is typically performed on the basis of knowledge of a shared secret.

More details are provided on message authentication in Chapter 2, so we will not go into any details in this introductory chapter. In short, this is how message authentication works: the sender provides proof for data integrity by running a so-called secret hash algorithm over the contents of the message and adds the results of the algorithm (called digest or hash) to the end of the message. Hash algorithms are mathematical one-way functions. In other words, while it may be straightforward to calculate the output of a hash function (digest) from an input data packet, it is extremely hard to determine what input packet has been used to create the output digest. However, hash algorithms are rather well known, and hence if no 
secrets are used, it is possible for an attacker to change the packet in flight and re-calculate a new digest and then to replace both the original packet and digest with the altered packet and the new digest. In order to prevent the MITM from running the same algorithm over an altered version of the message, the sender uses a secret when creating the hash. The secret is only known to the sender and the receiver. This process is often referred to as running a secret hash algorithm, even though the algorithm itself is usually known. Once the receiver receives the message, it runs the same hash algorithm over the same portion of the data and compares the result of its calculation with the hash provided by the sender and if there is a match, the receiver considers the data authentic, otherwise the receiver needs to discard the data.

The secret hash algorithms are examples of symmetric key authentication methods. Another method of providing message authentication is to create the so-called digital signatures. Digital signatures are typically based on public key cryptography. We will go through the details of these procedures in Chapters 2 and 3.

\subsubsection{Mutual Authentication}

We finalize the description of various authentication concepts with a short note on mutual authentication. Client authentication that was explained earlier is a unilateral authentication, where only one end of a communications channel proves that the identity it has presented to the other end is authentic. It would make sense that when establishing communications both ends authenticate to each other. However, the reason this type of unilateral authentication is so widespread is that in many cases, the client simply trusted the network. So the network does not have to prove to the client that it is also authentic. In the earlier cellular phone example, due to the large expense involved with setting up a cellular network, it would be hard to imagine that attackers will go through the trouble of setting up a whole network and its high towers simply to hijack a cellular phone subscription. For those reasons until very recently, neither the device nor the user required any authentication from the network.

With a proliferation of large number of access providers competing for customers, a user may be confronted with a number of competing networks with which she does not have any business or trust relationships. Take a mom and pop coffee shop for example: a customer entering the coffee shop does not know whether the mom and pop that own a coffee shop actually know how to operate a wireless local access network (WLAN) access point (AP) or how to protect their access points from being loaded with viruses and Trojan horses. For all a user knows, another customer may have found the WLAN AP behind the coffee grinder machine, disconnected it and simply installed another AP to re-route all the traffic in the shop to some place else, or simply passively copy traffic to a server. Imagine, if the customer was planning to do some Internet shopping while sipping coffee and was entering her credit card number at an e-commerce web site not using proper encryption methods. In this case, it makes sense for the coffee shop AP to authenticate itself to the user's device as well. As we can see the unilateral client authentication is not sufficient and has to be upgraded to a mutual authentication where the server also authenticates to the client.

The mutual authentication between the client and the server is a special case of a more generic case of mutual authentication, where the two parties are simply peers as opposed to client and server. In that case, each peer authenticates to the other, either sequentially, or in parallel. 


\subsubsection{Models for Authentication Messaging}

\subsubsection{Two-Party Authentication Model}

This model is used when the two peers interact with each other through a direct line of communication without the involvement of any middle nodes such as gateways or proxies. In such cases, the two entities directly authenticate to each other. The most prominent example is a direct client-server interaction, during which the client has to authenticate unilaterally to the server to gain access to service. However, mutual authentication can also be performed in direct two-party manner. As we will see later on, many key exchange mechanisms, such as Internet Key Exchange (IKE), require a direct two-party authentication exchange.

\subsubsection{Three-Party Authentication Model}

With the increase in size of networks and number of users wishing to access the network and its services, the networks have started to deploy specific points of presence (POP), which are low-cost unsophisticated devices without large processing power or database capabilities. The POPs typically interact directly with the users, but refer to central internal servers for many tasks and decisions regarding interactions with users. One such task is authentication. The POPs are typically incapable of authentication processing. Therefore, the two-party authentication model has been expanded to include three parties:

1. The supplicant, the user trying to gain access. In case of dialup networks, the user is configured with a phone number for the ISP modem pool and is given a password. The user dials the number of the modem pool and reaches one of the modems.

2. Authenticator, at edge interacting with user. In case of dialup, the authenticator is at the modem pool. The authenticator has no authority by itself, and is like the security guard at the door of a corporate building. When a visitor, who has no badge, walks in, the guard needs to call the authorities to ask whether it should let the visitor in or not. As we will see later on, in the AAA model, this entity is called the network access server (NAS), which acts as a AAA client.

3. Authentication server, who has the real authority and the necessary information database (e.g. a list of who is authorized, user names, and passwords) to make decisions regarding granting access to the user. In the security guard analogy, this is the boss upstairs that the guard calls to and the one who makes the ultimate decision. The AAA server in the AAA model is shown in Figure 1.1.

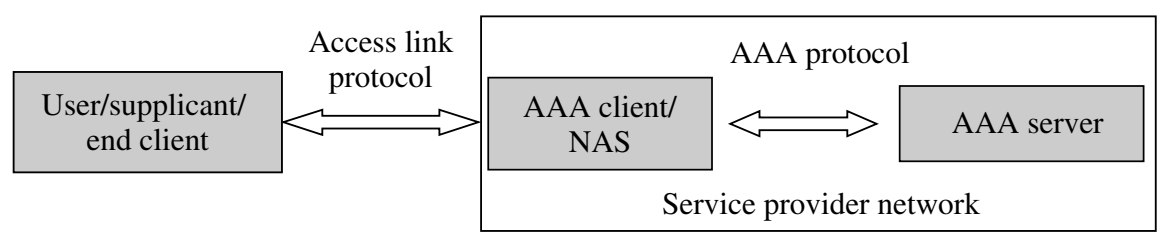

Figure 1.1 The three-party authentication model deploying an AAA infrastructure 


\subsubsection{AAA Protocols for Authentication Messaging}

In small networks, an authentication server could be configured by the system administrator at the authenticator, so the authenticator and authentication server are co-located. However, as we mentioned earlier, in large networks or multi-domain networks, this is not practical, and many network points of presence, acting as NASs are deployed and the authentication must happen according to the three-party model. Naturally, the end-to-end authentication exchange needs to happen between the supplicant and the AAA server, but the NAS is also involved in the exchange of authentication-related messaging. Since the NAS controls communications into and out of the private network, it needs to intercept messaging between the supplicant and AAA server. Furthermore, the NAS typically acts as a protocol dividing point, since the communications to the AAA server side of the NAS typically happens over a private and typically wired and trusted part of the network, while the communications on supplicant-side of the NAS is over an untrusted and many times wireless medium. In order to provide interoperability between various network equipments, protocols for various segments of the three-party model have been standardized. The protocols for communications between the NAS and the supplicant typically depend on the type of access technology that the network provider offers and many times are at a lower layer (link layer). However, the NAS communicates with the central authentication server over standard UDP/IP or TCP/IP protocols and can use a standard protocol for carrying authentication messages on behalf of the supplicant and the server. As we will see later on, experience has shown that the authentication server can be co-located with entities performing authorizations and accounting as well (a AAA server). For that reason, the protocol between NAS and the authentication server is now a AAA protocol as follows.

\subsubsection{User-AAA Server}

As mentioned earlier, there is no direct communication between user and AAA server. The most prominent AAA protocol today, RADIUS (stands for remote access dial in user service), was designed to allow a NAS forward a user's request and its credentials to the server, and then carry the server's response back to the user. The Access-Request, Access-Challenge message structure in RADIUS shows that it was designed to accommodate password-based authentication methods, so that NAS can forward an authentication request message from the user to the authentication server and issue eventual challenges created by the network and present to the user.

\subsubsection{NAS-AAA Server Communications}

As mentioned earlier, this communication is typically on a private part of network. A AAA protocol, such as RADIUS protocol, is used for this purpose. Original assumption was that there is only one hop between the NAS and the AAA server. However, multiple hops deploying AAA proxies may be required. We will discuss AAA proxies in Chapters 6 and 7. It is important to note that when proxies are involved, the NAS-AAA server communication may no longer be over a private network. This means the information carried between the NAS and AAA server over the AAA protocol may need special security protection. 


\subsubsection{Supplicant (User)-NAS Communications}

This communication is typically over a single-hop link called access link, since it is part of an access network. The access link provides a physical channel and a link layer protocol. The physical channel only provides the coding and modulation of information bits into electrical signals. Neither the earlier phone line dialup connections nor the later cellular systems provided layer- 2 services, such as packet formatting, framing, and multiple-access mechanisms, and required specific layer-2 protocols such as point-to-point protocol (described in Chapter 2) for this purpose. The new wireless access technologies such as 802.11 WLAN have their own framing mechanisms and do not need additional protocols such as PPP for carrying layer-2 level messaging. However, the main point is that as we mentioned before, the IP-level service should be established after the initial authentication and hence, the user-NAS exchange of authentication messages need to run directly over an access technology-specific layer-2 protocol.

\subsection{Authorization}

Authorization is defined as the act of determining whether a particular privilege can be granted to the presenter of a particular credential. The privilege can be right of access to a resource, such as a communications link, an information database, a computing machine, or many other things owned by a network or service provider. The presenter of the credential can be either a device or a user.

\subsubsection{How is it Different from Authentication?}

The problem of authorization and its distinctions from the problem of authentication can be easily explained with the following example. Let us assume that a person, holding a personal handheld device such as a wireless-link enabled PDA, has subscribed with a highpriced network operator. This person requests to see some movie clips on his personal device. She uses her personal device to connect to a wireless network, and based on the credentials that the network provider has given to her at the time of subscription, she can authenticate through her PDA and connect to the network. In many of the networks today, this authentication would be enough for her to access the movie clips from a server located inside the operator's network. However, imagine if the network provider would charge different prices for different movies or download speeds. A lower paying user is allowed to download the clips at a much slower speed. The user may request a higher quality of service (QoS) by agreeing to pay a one-time premium. In this case, a mere verification of user identity would not be enough for granting the service requested by the user. The network must somehow access the user profile, consult entities controlling the amount of available bandwidth, and then make a decision on whether or not to authorize the user to access the service.

Another commercial example where authorization is important is networks that provide service to users that have purchased pre-paid cards. A cellular phone user buys a pre-paid card that is supposed to allow her to make phone calls for three hours. Every time such user requests to make a phone call, the network must check to see whether there is any credit left on the user's card before allowing the user to connect.

In commercial applications, the problem of authorization usually either translates to protection of revenue or to exercising the right to a service. However, in public safety, 
military, and security applications, the consequences may be more severe. A low ranking officer should not be authorized to join a conference call held between army generals. Public safety responders may have special units dealing with specific emergencies, such as high-rise building fires. Dispatch calls or conversations within the unit need to remain private within the group, both for privacy issues and to save bandwidth. A fire fighter who is not part of a special unit should not be allowed to join the channel reserved for that special unit. In such a case, once the fire fighter and his device is authenticated to the network, the network must pursue with the act of authorization, i.e. check his profile against what is required to authorize an agent to join specific calls on specific channels.

Historically, a private enterprise owning the computer or radio networks simply authorized its employees or affiliates for use of its resource as long as they could prove their affiliation to the enterprise. The credentials for proof of affiliation were the authentication credentials that were given to the user at the time of initiation with the enterprise (as described in section 1.1 before). Once the user was authenticated, she was also authorized for service, in other words not only the authorization was equated with authentication, but also the credentials presented for authentication were also used for authorization. This model still exists in many enterprise networks: the second A (authorization) is coupled with the first A (authentication) in AAA. In commercial networks, authorization is based on acquisition of revenue, i.e. if the network provider knows it can collect payment from the user of the service, the user will be authorized for service. Here the second A is coupled with the third A (accounting). The user subscribes for a service and agrees to pay a fee for the service and the network provider agrees to provide the user with the service. However, even though we said that authorization is coupled with accounting and billing, this is only in theory (business agreement paper work). In practice, the network operator implements this agreement by giving the user the authentication credentials and again bases authorization on authentication.

Now that we have established that there is a difference between authentication and authorization, let us discuss another extreme example from real life, where authorization is actually more important than authentication. When we want to go see a movie at a movie theatre, we will pay the cashier and buy a ticket. Let us assume we pay cash and we are adults that are not bound by the viewing rating; once we receive the ticket, that ticket alone is all that we need to go to a hall and see the movie. The ticket is the credential needed to authorize us to see the movie and hence is a perfect example of authorization credentials. The movie ticket cannot be used instead of a passport to get onto an international flight. In other words, it is completely worthless as authentication credential (to verify our identity).

The networking counterpart of the movie ticket is the pre-paid calling card that is becoming popular. The pre-paid card allows the card holder to make a phone call through a network provider's infrastructure and the provider does not care who the user is as long as there is still credit left on the card.

\subsubsection{Administration Domain and Relationships with the User}

At this point, it is useful to also describe the concept of administrative domain: According to RFC 1136 [ADMRT1136], an administrative domain is "a collection of end systems, intermediate systems and sub-networks operated by a single organization or administrative authority". In AAA language, this typically means that the domain is served by the same AAA server (or pool of synchronized AAA servers, if failure recovery is important) and is 
ruled by the same network policies. When a user affiliates with a private network or subscribes with a commercial network provider, it is said to belong to the administrative domain. The AAA server within the domain is said to act as the user's home AAA server (AAAH or HAAA). The user's information and credentials are then stored at that AAA server and the user is said to have a trust relationship (and business relationship for commercial networks) with the administrative domain and its AAA server.

In a more general case, a user may roam to a network that is not part of its home administrative domain. The user may request services from this visited domain, which does not have any trust relationship with the user. In this general case, the service equipment, providing the service to the user, is part of a different administrative domain than the user's home domain and the visited network cannot grant any services to the user unless it has some relationship with the user's home domain. Two kinds of relationships exist between the user and the network (or between networks):

1. Contractual relationships: The user must have a contractual relationship with the service domain it is trying to connect to. The contractual relationship of the user is not with a specific network entity, but the entire domain. The authorization to obtain bandwidth or QoS is always processed on the basis of existing contractual relationship.

2. Trust relationship: The trust relationship is required for authentication or securing the communications between any two entities. In contrast to contractual relationship, the trust relationship is between the user and a specific component of the network not just with the entire domain. The trust relationship is usually instantiated in the form of a security association, which defined a set of pre-defined security algorithms and related keys to use to provide communications security.

Note that a contractual relationship is independent of trust relationship.

\subsubsection{Standardization of Authorization Procedures}

One can safely say that the second "A" (authorization) of the three "A"s within "AAA" is the one to which the least attention is paid in the standards developing organizations, such as Internet Engineering Task Force (IETF). Lack of attention may be something that most middle siblings in a large family of children complain about. Authorization has received its share of hand-me-downs from its bigger sibling, authentication: as we mentioned before, most of the time authorization is performed on the basis of same credentials as those used for authentication.

Realizing that distinction of authentication and authorization deserves a focused research and standardization effort, the IETF AAA working group formed a separate subgroup called authorization subgroup to start looking at procedures and standardization for authorization. However, eventually this activity gave way to more hard pressing issues in the AAA working group and was moved into a research group in Internet Research Task Force (IRTF), called AAA architecture group (AAAArch). The AAAArch group worked on several informational documents describing framework [AUTHFR2904], requirement [AUTHREQ2906], and application examples [AUTHAPP2905] for authorization. In this section, we give a few highlights from those specifications. However, the fact that these specifications come from the research branch of IETF (IRTF) rather than its engineering branch attests to the fact that these specifications have not caught on in the real world yet. Realizing that equating authentication 
and authorization and their credentials is becoming an issue, the IETF steering group (IESG) has recently urged network and AAA procedure designer within the engineering teams to start decoupling the authentication and authorization procedures through using a variety of tools such as separating authentication and authorization credentials. We have yet to see any significant progress in this area, but feel that it is important to recite the more important results of the IRTF documentations in the following.

A simplified architecture for systems implementing authorization mechanisms is shown in Figure 1.2. Upon receiving the request for a service or a resource, the service provider network first consults an authorization server that holds user profiles and can then make a determination on whether the user is authorized to use the service it has requested or not. The IETF authorization model reasonably assumes that authorization requests are only processed for authenticated users. In other words, we do not need to perform authorization in case we have not been able to successfully verify the end party's identity with certainty. Still, it would make sense that the same server performs both authentication and authorization, even though this server may fetch authentication credentials and user profile information from different databases. As we will see later on, since an accounting server also collects user's resource usage information, accounting is typically also performed by the same server, namely the AAA server: authentication, authorization, accounting server. The model also includes an entity that provides the actual service and is called the service equipment. The service can be something that the user considers as a service or something that provides some functionality within the network. Example of the first type of service is a music stream or a conference call, supported by an application server. In this case, the service equipment is the application server. Network functionality types of service are usually transparent to the user. For instance, a mobility agent is a service equipment that provides mobility services for the user's traffic.

The model shown here assumes that service equipment is administered by the same service provider network as the one that the user is subscribed to. In a simple case, typically called single administrative domain case, the service equipment is also part of the home administrative domain and can directly interact with the home AAA server of the user. In more general cases, the service equipment and the service provider network may be different from user's home domain.

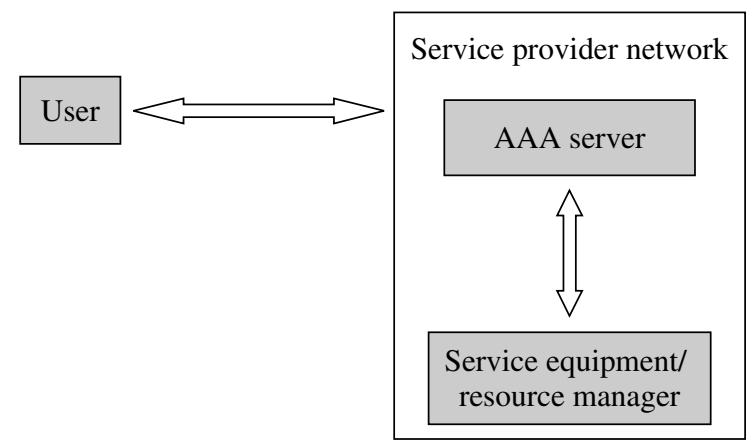

Figure 1.2 Service authorization model 


\subsubsection{Authorization Messaging}

Authorization of the user for service is accomplished on the basis of interaction between the user, the AAA server, and the service equipment. Three major scenarios are recognized for this interaction, each of which leads to a different sequence for the order in which the operations are performed:

- Agent sequence: This sequence is used for scenarios where the user contacts an entity in the AAA infrastructure first. The contact point is typically an edge entity that is also an AAA client. The user sends its service request, which can be seen as authorization request, towards the AAA server. The AAA server authorizes the user based on the information it has on the user or after consulting other entities such as a policy server or a resource manager, such as a bandwidth manager. After the authorization is successful, the AAA server sends the authorization possibly along with other configuration information to the service equipment. The service equipment prepares itself (e.g. sets up states and so on) for providing the service and possibly acknowledges to the AAA server that it has completed the configuration procedure. The AAA server replies to the user that authorization is complete and service is set up. Here, the AAA server acts as an agent for the user, and hence the sequence of events is called agent sequence.

- Pull sequence: In this scenario, the user sends the request directly to the service equipment. One example is, as we will see later on, when a user requests to use the services of a Mobile IP agent for support of her mobility. The service equipment forwards the request to domain's AAA server. Note that, in case, the user belongs to a different administrative domain, this AAA server is a local AAA server that must contact the user's home AAA server. The home AAA server evaluates the request and returns a response that eventually gets back to the service equipment. The service equipment accepts or rejects the service based on the AAA server response and notifies the user accordingly.

- Push sequence: This model is the one most similar to the movie theatre ticket. The user gets a ticket or certificate from the service provider AAA server. Anytime the user requests a service from the service equipment, the user presents the ticket to the service equipment as a way to show that it has been authorized by the AAA server to access that service.

\subsubsection{Policy Framework and Authorization}

When we were discussing the differences between authorization and authentication, we mentioned that after verifying the identity of a user requesting a service, the network needs to check the user profile and make an authorization decision based on that profile. In order to keep the authorization process consistent and scalable, the decision is often made with the help of a pre-set policy. Since many types of policies, such as security policy, group affiliation policy, and roaming policy exist, having a policy framework in place is important. The policy framework defines various architecture elements such as a policy repository, policy decision points (PDP), and so on. Policy repository typically includes the following information: (1) available services, (2) resources about which authorization decisions can be made, (3) policy rules to make authorization decisions, and (4) authorization event log for cases when authorization may be conditioned on the log of some previous events that must have happened. 
The policy framework also defines the processes for managing and sharing the policy information with other entities in the network. We do not go into the details of the policy framework and refer the reader to the work done by the Policy Framework working group in IETF. However, it is important to know that the AAA server may need to interact with entities, that we simply call policy servers in order to make appropriate authorization decisions. This interaction must allow the AAA server to retrieve the policy and enforce it during the authorization process.

\subsection{Accounting}

The final "A" in "AAA" is for Accounting. Even though a majority of engineers believe the terms accounting and billing have the same meaning, accounting involves more than tracking a user's total number of phone call minutes or data packets. A variety of applications are defined for accounting:

- Auditing: The act of verifying the correctness of an invoice submitted by a service provider, or the conformance to usage policy, security guidelines, and so on.

- Cost allocation: With the convergence of telephony and data communications, there is increasing interest in understanding the cost structure associated to each of the telephony and data portions.

- Trend analysis: Typically used in forecasting future usage for the purpose of capacity planning.

Each of the applications above may be processed at a different logic management entity. But in general, accounting is concerned with collection of information on resource consumption at all or specific parts of the network. This information is generally referred to as accounting data or accounting metrics. Typically, the network device providing services to a user collects information about user's resource consumption according to the accounting application's needs.

The accounting data collected by the network device is then carried by the accounting protocols over to the management entities responsible for each accounting application. As will be explained later on, each of these different accounting applications may have varying security and reliability requirements from accounting protocols; thus it is difficult to devise a single accounting protocol that meets the needs of every application. The goal of accounting management is to provide a set of tools that can be used to meet the requirements of each application. In the following sections, we go through the details of accounting management architecture.

\subsubsection{Accounting Management Architecture}

The accounting management architecture specifies interactions between network devices and accounting servers and any possible billing servers. It also defines procedures for collecting usage data. In the following, we define each of the concepts and components of accounting management architecture. 
- Accounting metrics: The data collected by the network device and transferred to the accounting server via the accounting protocol.

- Charging: Charging derives non-monetary costs for accounting data based on service and customer-specific tariff parameters. Different cost metrics may be applied to the same accounting records.

- Accounting server: The entity that is responsible for processing the accounting data received from the network device. This processing may include summarization of fractions of accounting information (called interim accounting), elimination of duplicate data, or generation of session records. In order to reduce the volume of accounting data and the bandwidth required to accomplish the transfer, the processed accounting data can be submitted to a billing server. Such session records may be batched and compressed by the accounting server prior to submission to the billing server.

- Billing: Billing translates costs calculated by the charging into monetary units and generates a final bill for the customer. Billing policies define among others the type of bill, e.g. invoice or credit card charge, the form of the bill, e.g. itemized or not, and the times for the bills, e.g. weekly, monthly.

- Billing server: The entity that handles rating and invoice generation, but may also carry out auditing, cost allocation, trend analysis, or capacity planning functions.

- Accounting proxy: The entity that acts as both a client and a server. When a request is received from a client, the proxy acts as an AAA server. When the same request needs to be forwarded to another AAA entity, the proxy acts as an AAA client.

- Local proxy: A AAA server that satisfies the definition of a proxy and exists within the same administrative domain as the network device (e.g., NAS) that issued the AAA request. Typically, a local proxy will enforce local policies prior to forwarding responses to the network devices, and are generally used to multiplex AAA message from a large number of network devices.

\subsubsection{Accounting Across Administrative Domains}

In networks with a large amount of users, large geographical footprint or in cases where users roam between networks owned by different service providers, the administration of each network can be managed by a separate accounting and billing server.

- Intra-domain accounting involves the collection of information on resource usage and it's processing within one administrative domain. In intra-domain accounting, accounting packets and session records typically do not cross administrative boundaries.

- Inter-domain accounting involves the collection of information on resource usage within an administrative domain, for use within another administrative domain. In inter-domain accounting, accounting packets and session records will typically cross administrative boundaries.

The architecture shown in Figure 1.3 depicts interaction between various entities within a multi-domain accounting model. The accounting server needs to distinguish between interand intra-domain accounting events and route them appropriately. A specific type of entity identifier, called network access identifier (NAI) is generally used for this purpose. The NAI consists of a user identity followed by a realm or domain identity in the form of userID@realmID. If the session record contains the NAI for the end node, the involved server can by examining the domain (realm) portion of the NAI identify the domain to which 


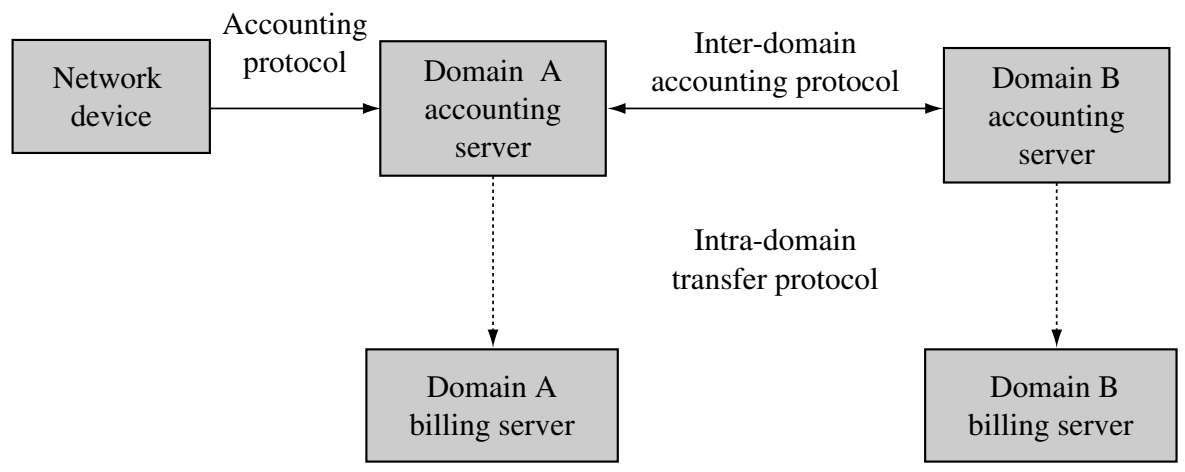

Figure 1.3 An illustration of accounting management architecture

the data need to be routed to. If the domain portion is absent or corresponds to the local domain, then the session record is treated as an intra-domain accounting event. Intra-domain accounting events are typically routed to the local billing server, while inter-domain accounting events will be routed to accounting servers operating within other administrative domains.

\subsubsection{Models for Collection of Accounting Data}

Several accounting data collection methods are implemented in the industry. Examples are the polling model and the event-driven model. In the following sections we provide a short description for a few of these models.

\subsubsection{Polling Models for Accounting}

In the polling model, an accounting manager will poll devices for accounting information at regular intervals. In order to ensure against loss of data, the polling interval will need to be shorter than the maximum time that accounting data can be stored on the polled device.

Without non-volatile storage, the polling model may result in loss of accounting data in case the device reboots. The polling model performs poorly in implementation of roaming applications, i.e. in scenarios where the accounting data may be collected at multiple network devices due to user's roaming. For example, if a roaming user is receiving services in a network other than its home domain, to allow issuance of a single bill to the customer, the foreign domain needs to have roaming agreement with the user's home domain and send the accounting data collected for the user to the user's home domain for processing. In order to retrieve accounting data for the user within a given domain, the accounting manager would need to periodically poll all devices in all domains, which would add a processing delay to the billing process, not to mention the routing problems that can arise.

\subsubsection{Event-Driven Models for Accounting}

In an event-driven model, a device will contact the accounting server when it is ready to transfer accounting data. Most event-driven accounting systems, such as RADIUS-based 
accounting systems, do not perform batching and hence transfer only one accounting event per packet. This model is called even-driven model without batching and is rather inefficient. An event-driven model typically stores accounting events that have not yet been delivered only until a timeout interval expires. Once the timeout interval has expired, the accounting event is lost, even if the device has sufficient buffer space to continue to store it. As a result, the event-driven model has the smallest memory requirement, and is the least reliable, since accounting data loss will occur due to device reboots, sustained packet loss, or network failures of duration greater than the timeout interval. The event-driven model is frequently used in networks with roaming applications, since this model sends data to the recipient domains without requiring them to poll a large number of devices.

Since the most basic event-driven model does not support batching, it permits accounting records to be sent with low processing delay, enabling application of fraud-prevention techniques. However, because roaming accounting events are frequently of high value, the poor reliability of this model is an issue. As a result, an event-driven polling model may be more appropriate.

In the event-driven model with batching, again, a device will contact the accounting server or manager when it is ready to transfer accounting data. However, the device contacts the server when a batch of a given size has been gathered or when data of a certain type are available or after a minimum time period have elapsed. Since, such systems transfer more than one accounting event per packet, they are more efficient. An event-driven system with batching will store accounting events that have not yet been delivered up to the limits of memory. As a result, accounting data loss will occur due to device reboots, but not due to packet loss or network failures of short duration. Note that while transfer efficiency will increase with batch size, without non-volatile storage the potential data loss from a device reboot will also increase.

Through implementation of a scheduling algorithm, event-driven systems with batching can deliver appropriate service to accounting events that require low processing delays. For example, high-value inter-domain accounting events could be sent immediately, thus enabling use of fraud-prevention techniques, while all other events would be batched. As a result, this approach can have good scalability and flexibility characteristics.

In the event-driven polling model, an accounting manager will poll the device for accounting data only when it receives an event. Events are typically generated by accounting clients. Examples of events include: when a batch of a given size has been gathered, when data of a certain type are available or lapse of a minimum time period. Without non-volatile storage, an event-driven polling model will lose data due to device reboots, but is resistant to packet loss, or network partitions of short duration due to its buffering nature. Unless a minimum delivery interval is set, event-driven polling systems are not useful in monitoring of device health.

The event-driven polling model can be suitable for use in roaming, since it permits accounting data to be sent to the roaming partners with low processing delay. At the same time, non-roaming accounting can be handled via more efficient polling techniques, thereby providing the best of both worlds.

Where batching can be implemented, the state required in event-driven polling can be reduced to scale with the number of active devices. Note that processing delay in this approach is higher than in event-driven accounting with batching since at least two round-trips are required to deliver data: one for the event notification, and one for the resulting poll. 


\subsubsection{Accounting Security}

In an accounting framework, two types of data communications are required: the exchange of accounting policies and the collection of accounting records. Both communications introduce potential security hazards. However, accounting records provide the basis for billing. Therefore, the motives and potential for fraud is extremely high in the collection of accounting data. Thus, different accounting applications may impose different requirements on security of accounting protocol. In this section, we describe security requirements for accounting protocols:

- Secrecy of accounting policies and accounting data: Unauthorized entities should not be able to read or modify accounting policies or accounting records.

- Authentication of accounting data and accounting policy sources: One should ensure that the data are originated from the original source. Source-authentication can be achieved by using digital signatures.

- Protection of the integrity of accounting policies and records: It should be ensured that the data was not modified on the way from sender to receiver. Data-authentication can also be achieved with digital signatures.

- Verification of generated accounting data for correctness: Correctness of accounting data generated by a service provider must be ensured. A provider may generate incorrect accounting records either deliberately or unintentionally through faulty configuration. These incorrect accounting records probably have the consequence of incorrect bills. Customers can verify the correctness of the accounting data through their measurements and/or through data collected by a trusted third party. A trusted third party can be an independent accounting service provider or a more general entity providing an auditing service.

\subsubsection{Accounting Reliability}

In this section, we describe reliability requirements for accounting protocols. Typically, accounting faults include packet loss, accounting server failures, network failures, and server reboots, and it is important that accounting management systems be scalable and reliable. However, different accounting applications may impose different requirements on accounting protocol. In applications such as usage-sensitive billing, cost allocation, and auditing, loss of accounting data can translate to revenue loss and there is an incentive to engineer a high degree of fault resilience. Some of the application-specific reliability requirements are listed below.

- Billing: When accounting data are used for billing purposes, the requirements depend on whether the billing process is usage-sensitive or not. Non-usage-sensitive billing does not require usage information; in theory, all accounting data can be lost without affecting the billing process. This would, however, affect other tasks such as trend analysis, auditing, and data on wholesale information. Usage-sensitive billing processes depend on usage information; therefore, packet loss may translate directly to revenue loss. As a result, the billing process may need to conform to financial reporting and legal requirements, and therefore an archival accounting approach may be needed. 
- Trend analysis and capacity planning: In trend analysis and capacity planning, the forecasts are inherently imperfect, high reliability is typically not required, and moderate packet loss can be tolerated.

\subsubsection{Interim Accounting}

Billing applications typically require the NAS to send messages to the AAA server, indicating the start and termination of sessions for which accounting is to be processed. However, the bulk of information, necessary for the billing process, such as session time, number of bytes transferred, etc. is only available in the messages that are sent at the end of session (such as accounting stop message). This means, if for some reasons, such as power failure, reboot, network problem, the NAS becomes unavailable for some time, it becomes impossible for a service provider to bill for the sessions initiated on that NAS.

A procedure called interim accounting provides a remedy to this problem by having the NAS sending periodic updates with information pertaining to the sessions to the AAA server. This way, interim accounting provides checkpoint information that can be used to reconstruct the session record in the event that the session summary information is lost. To accomplish this, a new accounting message called interim accounting message was introduced in [RADINTDR]. Interim accounting message is sent periodically from NAS to the AAA server. It should be noted that the applicability of interim accounting is limited. For instance, in case of packet loss due to network congestion, sending interim accounting data over the wire can make the problem worse by increasing bandwidth usage. Therefore, the use of on-the-wire interim accounting is best restricted to only long-lived sessions, where accounting data are of exceptionally high value. This is accomplished by simply choosing the interim-reporting interval to a value larger than the average session duration. This ensures that most sessions will not result in generation of interim accounting events, and the additional bandwidth as well as memory space consumed by interim accounting will be limited.

\subsubsection{Transport Protocols}

Accounting protocols dealing with session data need to be resilient against packet loss especially in inter-domain accounting, where packets often pass through network access points. Resilience against packet loss can be accomplished via implementation of a retry mechanism on top of UDP, or use of TCP or SCTP. UDP-based transport is frequently used in accounting applications. When implementing UDP retransmission, there are a number of issues to keep in mind: one of them is retransmission behavior, which is important that the retry timers relate to the round-trip time, so that retransmissions will not typically occur within the period in which acknowledgments may be expected to arrive.

\subsubsection{Fail-Over Mechanisms}

Congestion control is another issue where without non-volatile storage, the combination of congestive back off and buffer exhaustion can result in loss of accounting data. Accounting server fail-over is a powerful tool for providing resiliency against failures. In the event, a primary accounting server fails, it is desirable for the device to fail-over to a secondary server, which 
can take over the responsibilities of the primary device in a seamless manner. Providing one or more secondary servers can remove much of the risk of accounting server failure. Secondary fail-over servers have become commonplace.

\subsubsection{Prepaid Service: Authorization and Accounting in Harmony}

A new trend that has been emerging in the cellular telephony during the past few years is that the users instead of binding themselves to a single cellular operator through a longterm contract, simply buy a calling card for specified number of minutes. The pre-paid card allows the user to place phone calls up for a number of minutes up to the face value of the card through the network of any operator honoring the card. In the networking jargon, this is called pre-paid service. We consider pre-paid service as a perfect example where authorization and accounting go hand in hand. The user is authorized to make a call as long as there is credit available on her call. As the call proceeds, the accounting mechanism collects the usage information and informs a specific server, typically called pre-paid server to make sure that the amount of the credit on the card is depleted according to the usage information.

\subsection{Generic AAA Architecture}

We explained the three-party authentication model deploying a AAA server, and throughout this chapter attempted to show why authentication, authorization, and accounting procedures should be performed by the same server: the AAA server. In the remainder of this chapter, we complete this discussion by providing a brief overview of the generic AAA architecture defined by a former subgroup of the AAA working group in IETF. These activities later moved into the AAAArch group within IRTF.

The AAAArch team defined a generic AAA architecture described in the form of the experimental RFC 2903 [GENAAA2903], which specifies how the AAA architecture can interact with other network management entities. Each of these management entities provides a specific service or function, but since it receives assistance from the AAA infrastructure it is seen as an application for the AAA infrastructure (called AAA application). Examples of such services or applications are bandwidth management, quality of service, and mobility services. The fact that RFC 2903 is an experimental RFC indicates that this specification provides guidance (rather than a standard) for how such architectures can be designed in the future. As we will see in later chapters, early AAA protocols such as RADIUS were not designed on the basis of architecture in mind. However, newer protocols such as Diameter are designed this way. Diameter has separate specifications for each application, and this is why we feel paying some attention to the generic AAA architecture defined by RFC 2903 is worth the time. RFC 2903 defines a new concept called Application-Specific Module (ASM) to abstract the functionality of entity that the AAA server interacts with. The ASM is the entity that manages an application. Examples of ASMs are QoS managers, bandwidth brokers, and Mobile IP agents. RFC 2905 [AUTHAPP2905] provides more details on each application and its interactions with the AAA servers. The RFC 2903, however, by defining the concept of ASM, can define a generic interface between AAA servers and any type of management entity without getting into details of each application. 


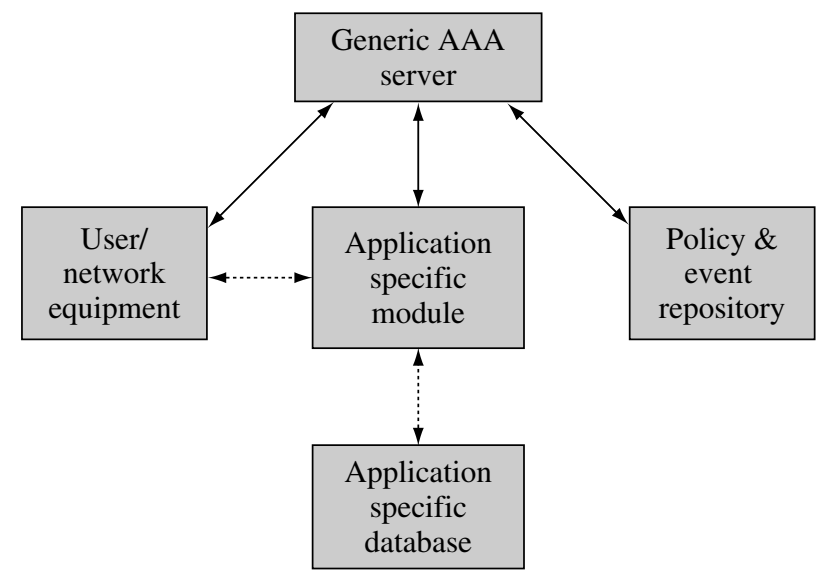

Figure 1.4 Generic model for interaction of AAA server with various management entities

Figure 1.4 shows the interaction between AAA server and other network entities according to the generic AAA architecture in RFC 2903. The authorization messaging process that we described earlier is an example of how such AAA architecture is deployed for authorization processes. As we saw, the details of the interaction between the user, AAA server, and application module depend on the specific deployment scenario and hence must be designed accordingly. The architecture, however, provides a framework for this interaction. The picture also shows that, besides the application-specific module, the AAA server may also need to interact with a policy repository to fetch the policy rules that apply to the user and the application at hand. The protocols used for interaction between the AAA server and the policy repository may be different from those used for interaction between the AAA server and the ASM. The same applies for interaction between ASM and its database (if this database is not co-located with the management entity itself).

It is important to note that each service has application-specific information (ASI), such as amount of bandwidth, that only the application-specific module (bandwidth broker in this case) understands. The AAA server that is supposed to authorize the request may not understand the details of each piece of information. Furthermore, the information may have a unique structure as well. Therefore, in general the AAA server is not required to have application-specific knowledge and needs to refer to the ASM, which was called service equipment during the discussion of authorization process earlier in this chapter. In order to divide the tasks between AAA server and ASM, the ASI needs to be separated from the logic needed for authorization, so the network equipments and AAA servers can treat the ASI in an opaque manner and send it back and forth as needed. All the AAA server needs to know is the location (ASM) to which it needs to send the application-specific data. As we will see later on, for most AAA protocols, specific data units called attributes are designed for each type of ASI. As long as the AAA can recognize the attribute type, it can simply tell what application it relates to. Typically, some sort of identifier for the ASM is included in the request to the AAA server, so that it can route the packet to the proper destination. For instance, an NAI may be used to help the AAA server identify the ASM or to let the AAA server know that the ASM may belong to a completely different administrative domain. 
The following provides an example on the chain of actions that should take place in the generic AAA architecture.

- The user or the network equipment that acts as an application agent for the user (such as an NAS) sends a combined authentication and service request to AAA server. For this request to be granted, the least required from the user is to present proper authentication and authorization credentials.

- AAA server verifies the authentication credentials for the user after consulting its user databases and possibly the policy repository. When authentication is successful, the AAA server inspects the contents of the authorization request and determines what the authorization is requested for.

- AAA server retrieves policy rules from the repository (if needed) and performs an authorization decision on each component (attribute) of the request according to one the following alternatives:

- When the component is an ASI that must be processed by the ASM, the component is sent to the ASM to be evaluated.

- Query the policy repository or an event log (if implemented) for an answer.

- Forward the component to another AAA server, if other administrative domains are involved.

- The AAA server informs the application-specific network equipment (service equipment) about the authorization and possibly provides necessary information for setting up the service at the point it is being delivered to the user (typically network point of presence), and if needed orders that POP to report accounting data back to the AAA server.

Two notes are in order: First, if any of the information components are to be maintained private and/or secure, the AAA server must make sure the components are encrypted and/or authenticated while in transit. Depending on the type of protocol used, message routing and security may be performed hop by hop or end to end as will be discussed in Chapters 6 and 7 . Second, the decision of authorization is still made by the AAA server, even if the services, resources, or policy decisions and management are performed at other entities. For instance, the AAA server decides whether the user is authorized to receive a high bandwidth data stream or a new temporary IP address, while the bandwidth broker or the mobile IP agent manage the admission control and bandwidth allocation or routing configurations, respectively.

\subsubsection{Requirements on AAA Protocols Running on NAS}

We discussed the role of network access server (NAS) in a three-party authentication model. However, since the NAS functionality is typically implemented inside network edge devices (network point of presence), the role of NAS has now changed from simply acting as a passthrough for authentication to a device that performs many access control and set up functions. Not only is the NAS a full blown AAA client that has a role in all the As in AAA (rather than just authentication), but also may support many different types of protocols such as access protocols, device management protocols, and routing protocols. So the general assumption is that the NAS must be able to support many of not all these protocols, and this makes the general perception of an NAS a rather non-standard one, since different network designers 
may expect different functionalities from the network edge devices. Hence, from an AAA standpoint, it is useful to define requirements on an NAS when it comes to support for AAA protocols. RFC 3169 [NASCRIT3169] has done just that. Both authentication, authorization, and accounting requirements are covered in this specification. In this final section of the chapter, we provide a few examples from this specification. It should be noted that these requirements are not specific for support of a specific AAA protocol; as a matter fact as we will see in Chapter 6, RADIUS clients (NAS) actually do not meet many of these requirements.

- AAA protocols are considered application level protocols and hence must be carried by other communications protocols, including transport layer protocols. Transport reliability requirement can be different for authentication messages and accounting messages. However, it is stated that AAA protocol must be able to detect a failure of the transport protocol to deliver a message within a known and controllable time period.

- In case of a AAA server failure, the AAA protocol must allow any sessions between an NAS and a given AAA server to fail-over to a secondary server without loss of state information.

- AAA protocol at NAS must support at least the RADIUS attribute set.

- The AAA protocol must provide mutual authentication between AAA server and NAS. At minimum, the AAA protocol should support use of shared secrets between NAS and AAA servers and avoid using the same shared secret for all NASs. More details on this will be provided in Chapter 6. The AAA protocol must also support public key infrastructure (PKI)-based methods for mutual verification of identity (authentication). We will discuss PKIs in Chapter 9.

- Some attributes (application specific information) may need to be encrypted. The AAA protocol must support selective encryption of attributes on an attribute-by-attribute basis.

- The NAS and AAA protocol must support transport (between NAS and AAA server) of credentials needed for authentication of users to servers and servers to users. Examples of credentials are password, response to challenges, X.509 certificates, and Kerberos tickets. Chapter 2 provides more detail on various authentication methods. The AAA must also provide means to hide these credentials from non-AAA entities during the transport.

- The NAS must be able to support multiphase authentication, such as prompting a text from NAS to user, engaging in challenge-response exchanges, and so on.

- The NAS and the AAA protocol must be able to support common types of identity, such as NAI and EAP identity request packets (see Chapter 2), and may be other network-specific identifiers.

- The AAA protocol must be able to provide an unambiguous location code that reflects the geographic location of NAS. It must also be able to provide unambiguous time stamp needed for authentication and auditing purposes.

- The AAA must provide means of assigning IP addresses to an incoming terminal as needed. The AAA protocol must provide a means of applying IP protocol filters to user sessions.

- For proper network operation management, it is important that the network load and usage is measured with accuracy, and for this purpose real-time accounting is important to monitor the status of sessions served by the NAS in real time. Therefore, the AAA protocol and NAS must support delivery of accounting information triggered by a number of events such as: start and end of sessions, expiration of certain pre-determined intervals, re-authorizations. 


\subsection{Conclusions and Further Resources}

This chapter attempted to present the basic problems as well procedures regarding to authentication, authorization, and accounting. Since the topic of authentication is a rather complicated one that has gone under tremendous amount of progress, we will devote another chapter on that topic. As mentioned earlier, authorization is less standardized. However, we expect that with the increase in user mobility and service offering, the field of authorization will go through much further progress. For now, most of the work related to authorization is categorized under policy frameworks. The reader is referred to the Policy Framework working group in IETF for further information in the policy area: http://ietf.org/html.charters/ policy-charter.html.

Also more information about authorization can be found in the AAA applications examples [AUTHAPP2905].

IETF documentations such as [ACCMGM2975] and [POLACC3334] can provide more information on accounting.

In the area of billing and accounting, many service operators may deploy their own specific technologies that follow their own policies. More information can be found from the companies providing AAA servers to these service operators. Examples are

- Bridgewater systems: http://www.bridgewatersystems.com/document_library/

- Interlink networks: http://www.interlinknetworks.com/resource/wp5-1.htm

- Funk software: http://www.funk.com/

\subsection{References}

1. [GENAAA2903], Laat, C. et al., "Generic AAA Architecture”, IETF, RFC 2903, August 2000.

2. [AUTHFR2904], Vollbrecht, J. et al., "AAA Authorization Framework”, IETF, RFC 2904, August 2000.

3. [AUTHREQ2906], Farrell, S. et al., "AAA Authorization Requirements", IETF, RFC 2906, August 2000.

4. [AUTHAPP2905], Vollbrecht, J. et al., "AAA Authorization Application Examples”, IETF, RFC 2905, August 2000.

5. [ADMRT1136], Hares, S. and Katz, D., "Administrative Domains and Routing Domains, A Model for Routing in the Internet”, IETF, RFC 1136, December 1989.

6. [ACCMGM2975], Aboba, B. et al., "Introduction to Accounting Management", RFC 2975, October 2000.

7. [RADINTDR], Calhoun, P. et al., "RADIUS Accounting Interim Accounting Record Extension", draft, January 1998.

8. [POLACC3334], Zseby, T. et al., "Policy Based Accounting”, RFC 3334, October 2002.

9. [NASCRIT3169], Beadles, M. and Mitton, D., "Criteria for Evaluating Network Access Server Protocols", IETF, RFC 3169, September 2001. 
